\title{
An Assessment of the Water Extraction Capabilities of the Heat Melt Compactor
}

Richard Alba ${ }^{1}$, Linden Harris ${ }^{2}$ and Kanapathipillai Wignarajah ${ }^{3}$

CSS-Dynamac Corporation, Moffett Field, California, 94035

John Fisher ${ }^{4}$

NASA Ames Research Center, Moffett Field, California, 94035

Mary Hummerick ${ }^{5}$

QNA-ESC, Kennedy Space Center, Fl 32899

Gregory Pace ${ }^{6}$

Lockheed Martin Space IS \& GS Civil, Moffett Field, California, 94035

Lance Delzeit ${ }^{7}$

NASA Ames Research Center, Moffett Field, California, 94035

and

\author{
Brian Larson ${ }^{8}$ \\ QNA-ESC, Kennedy Space Center, Fl 32899
}

The Heat Melt Compactor (HMC), a waste management technology developed at the NASA Ames Research Center, was designed to process waste generated aboard spacecraft. The device compacts, encapsulates and sterilizes the waste in preparation for onboard storage. In addition, the unit removes and recovers water, which is ultimately recycled, rendering the encapsulated waste inhospitable to microbial contaminants. Initial studies indicate that the HMC is capable of removing and capturing 90 to $98 \%$ of the water contained in the process waste sample. ${ }^{2}$ The nineteen experiments conducted at ARC described in this paper attempt to refine, quantify and define the limitations of the Heat Melt Compactor's dewatering and water collection capabilities. The amount of water in the initial waste sample was measured and found to be $19.04 \%$ by weight for batches made at ARC and $20.45 \%$ for those made at KSC. This was less than the percentage predicted from the standard waste model. The amount of water recovered and collected varied from 12.9 to $\mathbf{9 8 . 4 \%}$ of initial water contained in the waste. For the six tiles tested, the amount of water remaining in the tiles after processing ranged from 6.97 to $37.67 \%$. The water activity for five of these tiles averaged 0.472; all of these issues play a significant role in the survival and propagation of microorganisms. Water activity values below 0.6 inhibit microbial growth. Significant correlation was found to exist between Percent Water Recovery, Percent Expected Water Encapsulated in Tile and Water Activity, the latter two of which are inversely proportional to water recovered. Percent Water Recovery, since it is easily computed, can be used to predict the other two values.

\footnotetext{
${ }^{1}$ Engineer II, NASA ARC Code SCB, 239-15

${ }^{2}$ Scientist I, NASA ARC Code SCB, 239-15

${ }^{3}$ Senior Scientist, NASA ARC Code SCB, 239-15

${ }^{4}$ Lead Engineer, NASA ARC Code SCB, 239-15

${ }^{5}$ Research Scientist, NASA KSC M6-399, RM 3542G

${ }^{6}$ Engineer, NASA ARC Code SCB, 239-15

${ }^{7}$ Chemist, NASA ARC Code SCB, 239-15

${ }^{8}$ Mechanical Technician, NASA KSC, M6-399, RM 3542K
} 


\section{Nomenclature}

$\begin{array}{ll}\text { ARC } & =\text { Ames Research Center } \\ a_{w} & =\text { water activity } \\ H M C & =\text { Heat Melt Compactor } \\ J S C & =\text { Johnson Space Center } \\ K S C & =\text { Kennedy Space Center } \\ L P M & =\text { liters per minute } \\ m L & =\text { milliliter } \\ \text { psia } & =\text { pounds per square inch absolute } \\ \text { Std dev } & =\text { standard deviation }\end{array}$

\section{Introduction}

The Heat Melt Compactor (HMC), a waste management technology initially developed at the NASA Ames Research Center, was designed to process waste aboard NASA spacecraft. The device is designed to compact, encapsulate and biologically stabilize the waste in preparation for onboard storage. During processing the waste is compressed and heated. Plastics, contained in the waste, melt and spread throughout the remaining waste components and when solidified, encapsulate them. In addition, the hardware removes water, further reducing the risk of microbial contamination. Once removed, the water is available for collection, either at point of source or by the spacecraft's primary water recovery system. Initial studies have indicated that the HMC is capable of removing 90 to $98 \%$ of the water contained in the processed waste ${ }^{2}$. The purposes of the experiments described in this paper are fivefold:

- To determine empirically the actual amount of water contained in the pre-process waste sample batches.

- To determine the amount of water remaining in the tiles produced as a consequence of the heating and compaction process.

- To establish the level of water activity in the tiles after processing.

- To correlate the percentage of water removed with the amount of water remaining in the tiles and the water activity.

- To evaluate benefits and/or disadvantages of operating the HMC hardware at high and low pressures, that is, subatmospheric ( 2psia) vs. near-atmospheric ( 14psia).

Water activity $\left(a_{w}\right)$ is a measure of the ratio of the water vapor pressure over a material to the water vapor pressure of pure water under the same conditions in the range of $0-1$; typically pure distilled water has an $\mathrm{a}_{\mathrm{w}}$ of one. Multiplying this ratio by a 100 gives us the equilibrium relative humidity that the material would produce if enclosed with air in a sealed container at constant temperature. A piece of cloth for example, whose water activity is 0.45 , would produce an equilibrium relative humidity of $45 \%$. Water activity is important because it can predict the growth of bacteria, yeasts and molds ${ }^{3}$. Growth of these organisms in materials with $\mathrm{a}_{\mathrm{w}}$ of 0.60 or less will not be supported.

One of the key operational parameters of the HMC is system pressure, which can impact both water removal and water recovery. The amount of water removed, as stated above, can be correlated inversely to water activity. Reduced water activity, in turn, reduces microbial activity within the product tile, diminishing possible health risk to crew. Water recovery is not a function of the HMC hardware but could be accomplished with an ancillary water condensing and collection system placed nearby or directly on the hardware itself. Alternatively water vapor could be released into the cabin atmosphere, from which it could be recovered by the onboard humidity condensate systems $^{1}$. In either case this important resource is made available for recycling. An obvious goal of HMC functionality is to maximize water removal capabilities.

Nineteen experiments were conducted on tiles produced from waste samples formulated as per the standard logistic waste model ${ }^{4}$. For five of these we have a complement of data documenting:

- The amount of water recovered from waste samples.

- The amount of water remaining in the tiles after processing.

- The water activity of these tiles. 


\section{Materials \& Methods}

All waste compaction experiments discussed in this paper were performed using the Generation 1 HMC located at NASA Ames Research Center (ARC). Tests conducted at ARC to determine the operational characteristics of the HMC and produce HMC tiles for testing utilized a logistics model designed at NASA Johnson Space Center ${ }^{4}$. This model simulates both qualitatively and quantitatively the composition of waste streams generated aboard spacecraft involved in NASA space missions, and is known as the "standard waste model." Fifteen of the experiments discussed in this paper were part of a microbial test series done in collaboration with NASA Kennedy Space Center (KSC). Waste batches used in these tests were prepared at KSC. The remaining four experiments discussed in this paper used batches prepared at ARC. Waste was processed at temperatures between $130-180^{\circ} \mathrm{C}$, and process times (time held at maximum temperature) between 50-194 min. The fifteen microbial samples were processed using a waste chamber pressure of $2 \mathrm{psia}$, and a sweep gas flow rate of 1 LPM. The four ARC-prepared samples were processed using a waste chamber pressure of 14 psia, and a sweep gas flow rate of 0.2 LPM. Water recovered from the waste was collected using sweep air and an ice bath condenser system. Sweep air from the lab flows through the plenum, a region surrounding the waste chamber that connects to it via dozens of open holes. Since the waste chamber is held under vacuum, steam generated by the waste during processing is drawn downstream where the water is condensed out using an ice bath and collected in a graduated cylinder.

The standard waste model provides water fraction estimates for each waste constituent, resulting in an estimated total batch water content of $27.24 \%$ by weight. Water recovery calculations made prior to this paper used that estimate for the expected water value. Although the waste model specifies waste batch constituents and quantities, water contents varied slightly based on product availability and preparation methods. Three batches were prepared using the same ingredients and methods used in preparing the batches for HMC experiments at ARC. For these batches, items composed of polyethylene and polyethylene terephthalate, which are known not to contain water, were omitted to prevent water vapor from being trapped within the melting plastic. Each batch was weighed and placed in a convection oven at $130^{\circ} \mathrm{C}$, and left to dry for a minimum of 24 hours. At the end of the drying cycle, each batch was removed from the oven, weighed and returned to the oven for another cycle. This process was repeated until no further weight loss occurred. Water content was calculated by dividing weight lost by the initial batch weight, where initial batch weight was considered to be the actual starting weight of the batch plus the weight of plastic contained in a standard batch.

Although ARC and KSC personnel used the same logistics model to prepare HMC batches, brands and preparation methods varied slightly for five batch constituents. All other items were determined to be prepared identically. The five items in question were dried individually as per the KSC preparation method and the ARC preparation method to obtain specific water fractions, which were then used to adjust the full batch water content obtained from the ARC tests, described above, to provide a full batch water content for batches prepared by KSC. Macaroni \& cheese was cooked during batch preparation by KSC, but not by ARC. ARC did not dry this item individually, as only dry noodles were used in batch preparation, which were assumed to have a water content of zero. The four remaining constituents in question (strawberries, spinach, peaches, and rice) were dried in a vacuum oven (with only a slight vacuum drawn and vent open to circulate air) at $70^{\circ} \mathrm{C}$ for six or seven days. Each item was dried by the ARC team and the KSC team using their respective brands and preparation methods. Samples at ARC were dried in triplicate, and one sample of each food item was dried at KSC. Based on the oven drying tests, water fractions were calculated for each item. The difference in water fraction between the ARC method and KSC method was multiplied by the weight of the given constituent in a standard batch, giving the difference in water mass between the two batch preparation methods. These results were used to adjust the water fraction in the ARCprepared batch to calculate the water fraction in a KSC-prepared batch.

Based on empirically determined water content of standard batches and water recovered from waste batches during HMC experiments, Percent Water Recovery was calculated for each HMC test. For tests that used batches prepared at KSC, the KSC total batch water content was used for the water recovery calculation. Thirteen of the fifteen KSC-prepared batches discussed in this paper contained a $15 \mathrm{~mL}$ solution of water containing microbes ${ }^{5}$. This portion of the batches was considered to have a water content of $100 \%$, and was taken into account when calculating the expected water content of KSC-prepared batches. For tests that processed batches prepared at ARC, the ARC total batch water content was used for the water recovery calculation.

Eight HMC tiles produced from KSC-prepared waste batches were analyzed for water activity using a $\mathrm{Pa}_{\mathrm{w}} \mathrm{kit}$ water activity meter by Decagon Devices (Pullman, Washington). Three core samples were removed from each tile using a hole borer and placed into the sample cup provided by the manufacturer. Readings were taken according to the user manual ${ }^{6}$. 
For each processed batch, there is a portion of water that is not recovered. Visual examination of the processed tiles indicated that they contained some amount of water. To determine how much water was contained in product tiles, six tiles were shredded and dried. Five of these tiles had been analyzed for water activity at KSC. These tiles had been processed at $140^{\circ} \mathrm{C}$ (three tiles), $150^{\circ} \mathrm{C}$, and $180^{\circ} \mathrm{C}$, at soak times ranging from $73-130$ minutes, and all at approximately 2 psia waste chamber pressure. The sixth tile was a product of an ARC-prepared batch and was processed at $150^{\circ} \mathrm{C}$ for a soak time of 73 minutes at a waste chamber pressure of $14 p s i a$. These six tiles were chosen based on availability, as many other tiles had been destroyed in data analysis processes unrelated to this paper. Each tile was weighed (Figure 1; A), then cut into pieces about 1" x 1" using a band saw (Figure 1; B). Those pieces were weighed, then ground using a dry mill (Glen Mills, Inc., Clifton, NJ) (Figure 1; C).

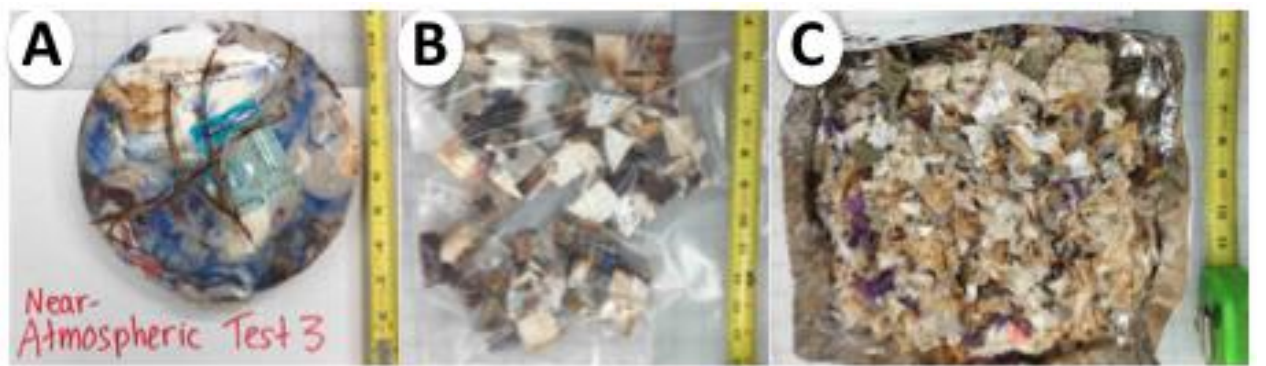

Figure 1. An HMC product tile (A) cut into 1" $x$ 1" pieces (B) using a band saw, then shredded using a dry mill $(C)$. compared to the initial weight of the shredded material to determine the amount of water contained in the tile. The initial weight of the tile was not used in the calculation because several grams were lost during the sawing and shredding process.

\section{Results}

The three ARC-prepared standard waste batches oven dried at $130^{\circ} \mathrm{C}$ had an average water content of $19.04 \%$ by weight $(\mathrm{n}=3$; std $\operatorname{dev}<0.001)$. Based on results from the individually dried food samples, KSC-prepared batches contain $7.07 \mathrm{~g}$ more water than ARC-prepared batches. Therefore a KSC-prepared batch has a water content of $20.45 \%$ by weight. Experimentally determined water contents were found to be lower than estimates provided in the Logistics Model (Figure 2).

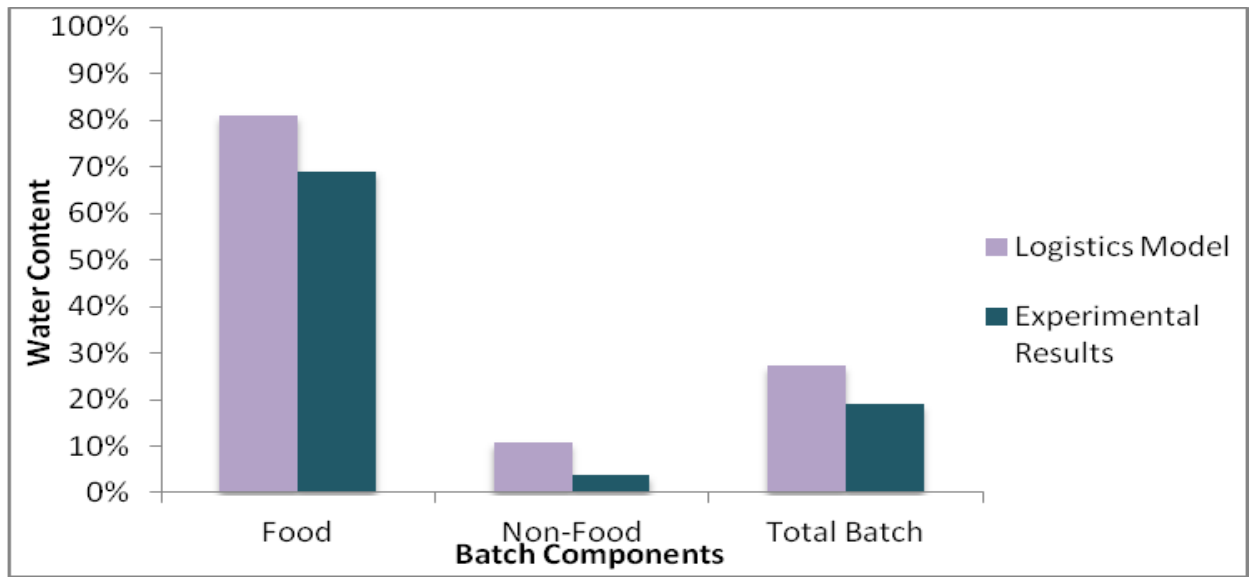

Figure 2. Comparison of standard batch water content estimates in Logistics Model and empirically determined batch water contents.
Water fractions calculated by oven drying experiments, taking into account the $15 \mathrm{~mL}$ inoculum in some of the KSC batches as discussed in the methods, were used to calculate the Expected Water and Percent Water Recovery data listed in Table 1.

Water Activity in eight tiles averaged 0.463 (std dev=0.054). Three of these tiles are not discussed further in this paper due to lack of water recovery data. The five tiles that were tested for water activity and also 
shredded for water content tests had water activities ranging from $0.423-0.550$ (Table 1).

The process of cutting tiles with a band saw caused a loss of $4.3-5.8 \mathrm{~g}$ per tile. The process of shredding pieces of tile caused a loss of $2.2-5.8 \mathrm{~g}$ per tile. The water content of HMC tiles processed under subatmospheric conditions ranged from $2.29-3.47 \%$. The tile processed under near-atmospheric conditions had a water content of $8.26 \%$. Note that only one near-atmospheric tile was available for testing. Water content of tiles was also compared to the expected water content of their respective batches. In the subatmospheric tiles, $6.97-10.97 \%$ of the batch water content was encapsulated in the tile. In the near-atmospheric tile, $37.67 \%$ of the batch water content was encapsulated in the tile.

Table 1. Process conditions and water recovery data for nineteen HMC experiments. Water Expected was calculated based on the $\mathbf{1 9 . 0 4 \%}$ batch water content determined empirically.

\begin{tabular}{|c|c|c|c|c|c|c|c|c|}
\hline Test \# & $\begin{array}{l}\text { Process } \\
\text { Temp } \\
\text { (C) }\end{array}$ & $\begin{array}{c}\text { Process } \\
\text { Pressure } \\
\text { (psia) }\end{array}$ & $\begin{array}{l}\text { Soak } \\
\text { Time } \\
\text { (min) }\end{array}$ & $\begin{array}{c}\text { Water } \\
\text { Recovered } \\
(\mathbf{m L})\end{array}$ & $\begin{array}{c}\text { Water } \\
\text { Expected } \\
(\mathrm{mL})\end{array}$ & $\begin{array}{l}\text { \% Water } \\
\text { Recovery }\end{array}$ & $\begin{array}{c}\text { \% Expected } \\
\text { Water } \\
\text { Encapsulated } \\
\text { in Tile }\end{array}$ & $\mathbf{a}_{\mathbf{w}}$ \\
\hline $3 \mathrm{M}$ & 180 & 2 & 180 & 108 & 117.2 & 92.1 & & \\
\hline 7M & 140 & 1.5 & 72 & 54 & 101.8 & 53.1 & & \\
\hline $8 \mathrm{M}$ & 140 & 1.5 & 72 & 72 & 100.9 & 71.3 & & \\
\hline $9 \mathrm{M}$ & 180 & 1.5 & 139 & 115 & 116.9 & 98.4 & & \\
\hline 10M & 180 & 1.5 & 129 & 110 & 116.5 & 94.4 & & \\
\hline $11 \mathrm{M}$ & 180 & 1.5 & 147 & 102 & 113.5 & 89.9 & & \\
\hline $12 \mathrm{M}$ & 180 & 1.5 & 129 & 86 & 114.0 & 75.5 & 7.28 & 0.450 \\
\hline 10DL & 130 & 2 & 69 & 107 & 113.6 & 94.2 & & \\
\hline 11DL & 140 & 2 & 74 & 106 & 113.6 & 93.3 & 7.4 & 0.423 \\
\hline 12DL & 140 & 2 & 130 & 70 & 113.6 & 61.6 & 7.92 & 0.467 \\
\hline 13DL & 140 & 2 & 74 & 60 & 114.0 & 52.6 & 10.97 & 0.550 \\
\hline 13M & 150 & 1.7 & 194 & 33 & 116.3 & 28.4 & & \\
\hline $14 \mathrm{M}$ & 150 & 1.7 & 193 & 45 & 115.0 & 39.1 & & \\
\hline $15 \mathrm{M}$ & 150 & 1.7 & 193 & 15 & 115.9 & 12.9 & & \\
\hline $16 \mathrm{M}$ & 150 & 2.5 & 82 & 77 & 116.3 & 66.2 & 6.97 & 0.47 \\
\hline Atmos1 & 180 & 14.2 & 84 & 74.3 & 92.6 & 80.2 & & \\
\hline Atmos2 & 180 & 14 & 50 & 69 & 93.8 & 73.6 & & \\
\hline Atmos3 & 150 & 14 & 73 & 24.5 & 95.6 & 25.6 & 37.67 & \\
\hline Atmos4 & 130 & 13.8 & 73 & 29 & 95.3 & 30.4 & & \\
\hline
\end{tabular}

None of the experiments achieved $100 \%$ water recovery. For six of the tests, we were able to determine how much of the unrecovered water had been encapsulated in the tiles (Table 1; Figure 3). All of the water in batch \# $11 \mathrm{DL}$ (subatmospheric, $140^{\circ} \mathrm{C}$ ) was accounted for when recovered water and water encapsulated in the tile were combined. Water encapsulated in the other five tiles tested could not account for the unrecovered water (Figure 3 ). 


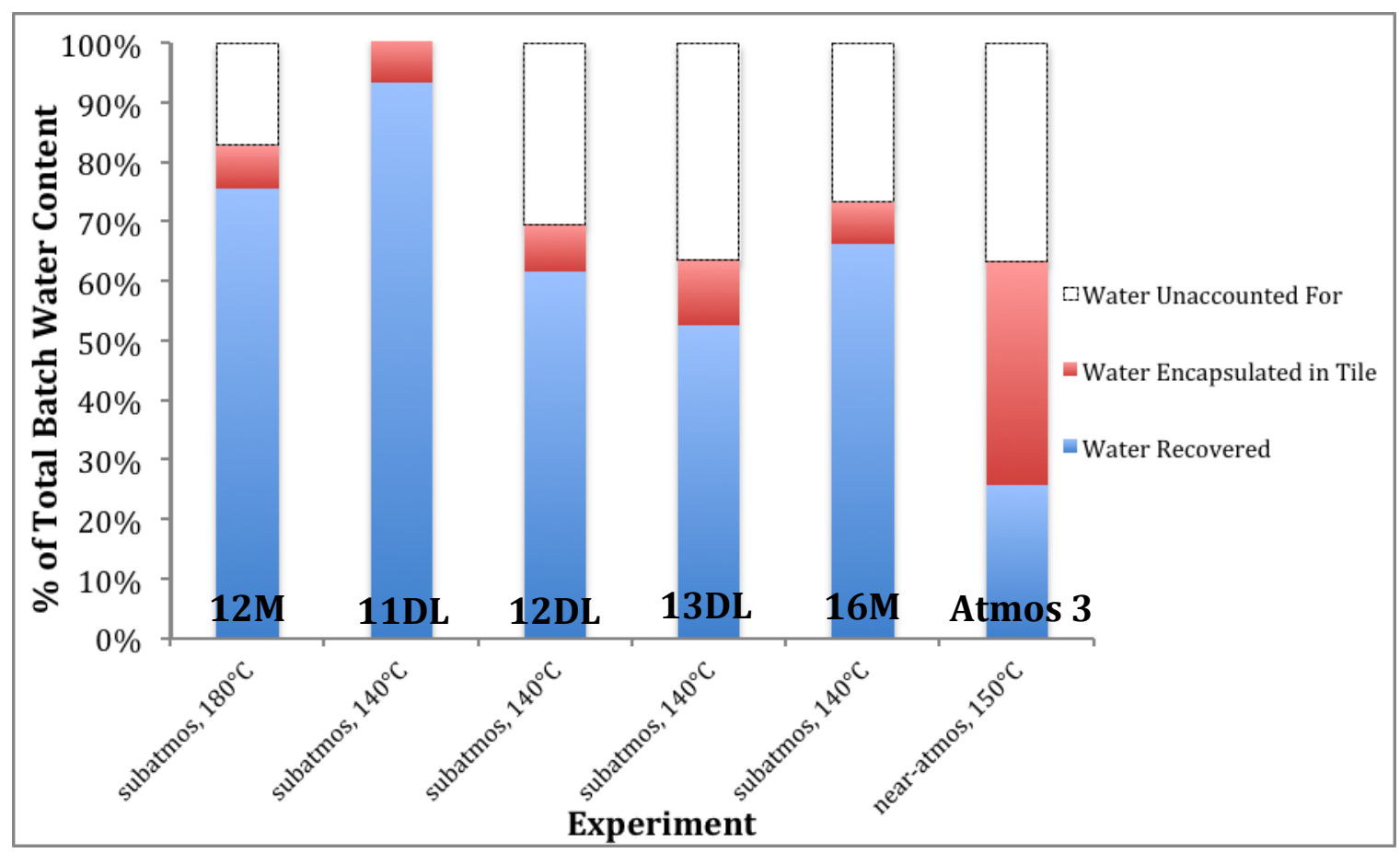

Figure 3. Of the initial batch water content, a certain percentage was recovered by the condensation system, a lesser percentage was left encapsulated in the product tile, and in most cases a certain amount of water was unaccounted for.

Three methods were used to assess water recovery of each experiment: Percent Water Recovery based on condensate collection, Percent of Expected Water Encapsulated in Tiles, and Water Activity. The results of these three methods correlated significantly with each other. When compared to Percent Water Recovery, Percent of Expected Water Encapsulated in Tiles yielded $R^{2}=0.87(n=6)$, and Water Activity yielded $R^{2}=0.76(n=5)($ Figure 4$)$.

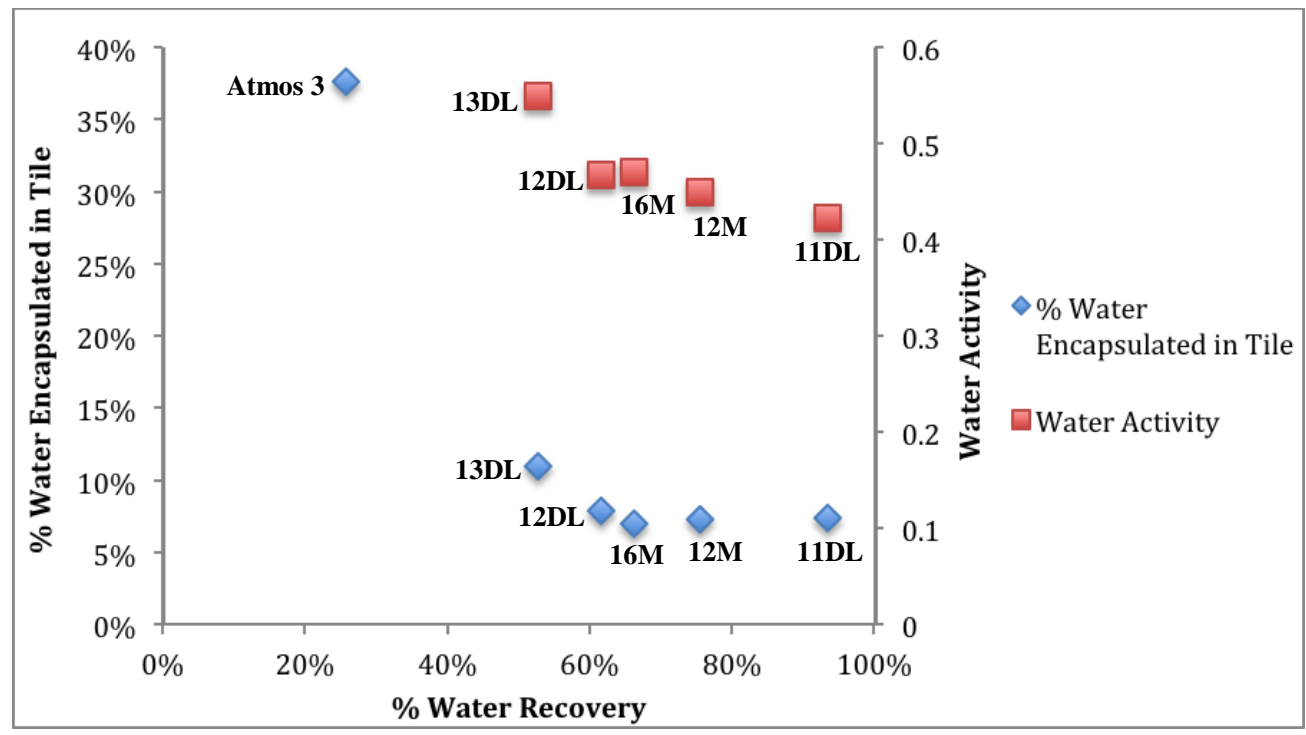

Figure 4. The relationship between Percent Expected Water Encapsulated in the Tile, Percent Water Recovery, and Water Activity. 


\section{Discussion}

The standard waste model provides water fraction estimates for each waste constituent, resulting in an estimated average batch water content of $27.24 \%$. The results of the oven drying tests conducted at ARC indicated an average water content of $19.04 \%$. The difference can be explained as follows. First, the predicted water content of the standard waste model is an estimate based on information taken from generic tables in a textbook ${ }^{7}$. The information presented represents average values from multiple sources and is, therefore, approximate. Variances from measured values are to be expected. Secondly although the waste model specifies waste batch constituents and quantities, the amount of water in each could vary depending on brand, storage conditions, and preparation. Note that product brand was specified in the logistics model, but specific brands were not always available during product procurement. For the purposes of this paper, we wanted water recovery data to be as precise as possible, and thus needed empirical data in order to provide an accurate total batch water content.

For the five tiles for which we had water activity data, the Percent Expected Water Encapsulated in Tile, that is, the percent of the water in the original unprocessed sample which remains in the processed tiles, was determined by the series of oven drying tests. Statistical analysis indicated significant correlation with Percent Water Recovery and with Water Activity (Figure 4). As a consequence, in future experiments, Percent Water Recovery can serve as a proxy indicator for the other two values because it is easier to measure.

The nineteen waste batches used in the experiments discussed in this paper all weighed approximately 500 grams; of this water comprised 19.04 to $20.45 \%$ by weight. The amount of water recovered ranged from as little as 12.9 to as high as $94.4 \%$ by weight. In all cases, a portion of the original water content was unaccounted for. Comparisons of the weights of the sample batches with the weights of their corresponding product tiles as well as data from the oven drying tests indicates that some of this water remains encapsulated in the tiles. Adding the amount of encapsulated water to the amount of water collected, however, still does not equal the total batch water content (see Figure 3). It has been unclear exactly what happened to this water but a number of explanations have been suggested. Over the course of its lifetime, the HMC hardware has been completely or partially dismantled a number of times. Examination of the various sub-assemblies revealed the presence of residues deposited on their internal surfaces ${ }^{8}$. For the most part these residues were either dry organics thought to be decomposition products of the sugary liquids contained in drink pouches or plastics, which passed though the holes in the compaction chamber walls, and into the plenum surrounding it. Some sections of this area however, particularly the floor, were covered with a residue that was somewhat sticky and could contain water, albeit in minor amounts. Another possibility is that water becomes trapped in the hardware plumbing. When flushing the system with a sweep gas after the hardware has been idle for a time after a test, water has been seen dripping from plumbing associated with the condenser, heat exchanger, and plenum exit. Another source of water loss involves the seals surrounding the restraint piston, which forms the bottom of the compaction chamber. In the past these seals have failed and water has leaked into the area below the chamber. Further investigation is clearly warranted in order to establish conclusively what is happening to the missing water.

As previously stated water activity is important because it can predict the growth of microorganisms, which could pose health risks to crew. Growth of these organisms is inhibited in material with an $\mathrm{a}_{\mathrm{w}}$ of 0.60 or less. Tests conducted at KSC verify that tiles produced by the HMC have an average $\mathrm{a}_{\mathrm{w}}$ of 0.463 and are therefore biologically stable. It must be pointed out however, that the water activity of any material is dependent on the environmental conditions to which it is exposed, particularly humidity and temperature. For example, tiles produced in an environment with a relative humidity of $34 \%$ and a temperature of $23^{\circ} \mathrm{C}$ will exhibit water activity that renders them biologically inert. Storing these tiles, however, in conditions of higher humidity or placing them on cold surfaces could change their $\mathrm{a}_{\mathrm{w}}$ to levels that support the growth of microorganisms. For this reason it may be necessary to contain processed tiles in a sealed environment prior to storage.

On the question of effect of operating pressure (subatmospheric vs. near-atmospheric) on water removal, our results are inconclusive. System degradation in late 2012 caused anomalously low water recovery on several subatmospheric tests, driving down the average water recovery of subatmospheric tests. Also, near-atmospheric tests must be replicated at consistent temperatures in order to make statistical comparisons of the results.

\section{Conclusion}

The purpose of this paper has been to discuss the series the tests conducted at ARC to characterize the performance of the first generation Heat Melt Compactor in terms of water extraction. The amount of water in the waste sample batches was established empirically, as was, the amount of water remaining in the product tile. This was correlated with water activity data generated at KSC. Process conditions and water recovery data was collated, 
reviewed and presented. Evaluation of the advantages of operating the HMC at atmospheric versus subatmospheric conditions was inconclusive due to lack of sufficient data. This investigation is ongoing.

\section{Acknowledgments}

Funding was provided by NASA Headquarters Advanced Exploration Systems Division.

\section{References}

${ }^{1}$ Wieland, P. O., Designing for Human Presence in Space: An Introduction to Environmental Control and Life Support Systems, NASA Reference Publication RP-1324, 1994.

${ }^{2}$ Pace, G. S., Fisher J.W., Delzeit, L., Alba, R.G., and Polansky, A., "Development of a Plastic Melt Waste Compactor for Human Space Exploration Misions - A Progress Report", International Conference on Environmental Systems 2010, AIAA.

${ }^{3}$ http://www.drinc.ucdavis.edu/dairychem4_new.htm [Cited April 07, 2014].

${ }^{4}$ Ewert, M. K., and Broyan, J. L., "Mission Benefits Analysis of Logistics Reduction Technologies", International Conference on Environmental Systems 2013, AIAA.

${ }^{5}$ Wheeler, R. L., Strayer, R. F., and Hummerick, M. E., "Microbial Characterization of Heat Melt Compaction for Treatment of Space Generated Solid Wastes: FY12 End of Year (EOY) Report for NASA Advances Exploration Systems; Logistics Reduction and Repurposing Grant. Heat Melt Compaction Task," KSC, NASA, John F. Kennedy Space Center, Florida, October 2012.

${ }^{6} \mathrm{http} / / / \mathrm{www}$. aqualab.com/products/water-activity-meters/pawkit/ [Cited March 28, 2014].

${ }^{7}$ Souci, S.W., Fachmann, w. and Krat, H., Food Composition and Nutrition Talbles, Medpharm Scientific Publishers, Stuttgart, Germany, 1994, ISBN 3-88763-027-0.

${ }^{8}$ Alba, R. G., Harris, L.C., Fisher, J., Wignarajah, K., and Pace, G. "Performance of the Gen 1 Heat Melt Compactor and Lessons Learned to Enable Further Engineering Development", International Conference on Environmental Systems 2013, AIAA. 